\title{
Childhood sleep duration modifies the polygenic risk for obesity in youth through leptin pathway: the Beijing Child and Adolescent Metabolic Syndrome cohort study
}

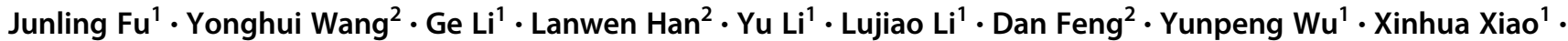 \\ Mingyao $\mathrm{Li}^{3} \mathbb{1}^{3} \cdot$ Struan F. A. Grant ${ }^{4,5,6} \cdot \operatorname{Ming~} \mathrm{Li} \mathbb{1}^{1} \cdot \operatorname{Shan} \mathrm{GaO}^{2}$
}

Received: 11 April 2018 / Revised: 10 May 2019 / Accepted: 19 May 2019 / Published online: 8 July 2019

(c) The Author(s) 2019. This article is published with open access

\begin{abstract}
Background/objectives Short sleep is an obesity risk factor, however, little is known about its interplay with genetic predisposition and pathways involved in obesity pathogenesis, especially in the longitudinal setting. We aimed to investigate a possible sleep-gene interaction for childhood obesity risk, and whether the interaction in childhood longitudinally contributes to obesity risk at a 10-year follow-up and further to test if there is any mediation through the leptin pathway.

Subjects/methods A total of 3211 children from China (6-18 years) at baseline and 848 participants at 10-year follow-up from the Beijing Child and Adolescent Metabolic Syndrome (BCAMS) cohort study were analyzed. Baseline leptin concentrations and 12 established adult body mass index (BMI) loci were examined for the associations with habitual sleep duration.

Results After adjusting for covariates, including pubertal stages and behavioral factors, short sleep duration at baseline was significantly associated with increased overweight/obesity risk at both baseline and follow-up. Genetic predisposition scores (GPS), particularly consisting of leptin-related SNPs $\left(\mathrm{GPS}_{\text {leptin }}\right.$ ), were robustly associated with baseline overweight/obesity in children who slept $\leq 8 \mathrm{~h} /$ day $(P<0.001)$, whereas the association was ablated in those who slept $\geq 10 \mathrm{~h} /$ day $(P>0.05)$. Comparable observations were made at follow-up. Mediation analysis revealed a modest direct effect of the $\mathrm{GPS}_{\text {leptin }}$-sleep interaction on BMI at baseline, while a significant indirect effect of this interaction was found to be mediated principally through elevated leptin (proportion: 52.6\%); moreover, the mediation effect via leptin remained stable over 10 years.

Conclusions This study suggests that shorter sleep duration in children from China $(<8 \mathrm{~h} /$ day $)$, compared to longer sleep duration ( $\geq 10 \mathrm{~h} /$ day), has a long-term impact on the association of polygenic risk for obesity from childhood to young adulthood and leptin pathway explains a key mechanism via a modification effect. Therefore, adequate sleep duration during childhood is important for the early prevention of obesity, especially if there is a genetic predisposition to this trait.
\end{abstract}

This study was registered at www.clinicaltrials.gov, and the Registration number is NCT03421444.

Supplementary information The online version of this article (https:// doi.org/10.1038/s41366-019-0405-1) contains supplementary material, which is available to authorized users.

Ming Li

liming@pumch.cn

$\triangle$ Shan Gao

gaoshanmw@163.com

1 Department of Endocrinology, NHC Key Laboratory of Endocrinology, Peking Union Medical College Hospital, Chinese Academy of Medical Sciences and Peking Union Medical College, 100730 Beijing, China

2 Department of Endocrinology, Beijing Chaoyang Hospital, Capital Medical University, 100043 Beijing, China

\section{Introduction}

Obesity rates have increased sharply over the past 20-30 years, particularly in childhood. The epidemic of obesity poses a major risk for the development of type 2 diabetes,

3 Department of Biostatistics and Epidemiology, University of Pennsylvania, Philadelphia, PA 19104, USA

4 Division of Endocrinology, The Children's Hospital of Philadelphia, Perelman School of Medicine, University of Pennsylvania, Philadelphia, PA 19104, USA

5 Division of Human Genetics, The Children's Hospital of Philadelphia Research Institute, Philadelphia, PA 19104, USA

6 Department of Pediatrics, Perelman School of Medicine, University of Pennsylvania, Philadelphia, PA 19104, USA 
cardiovascular disease, and certain types of cancer ${ }^{1}$. It is now known that both genetic and environmental factors contribute to the complex etiology of this trait. Among the changeable environmental factors that can influence risk, the obesogenic diet, and low physical activity are considered two major adult risk factors; however, other potentially plausible environmental factors, like short sleep duration, have been gaining increasing attention in recent years $[2,3]$.

Meta-analysis of cross-sectional [4] and longitudinal studies [2] showed that short sleep duration was associated with the risk of obesity. However, the mechanistic links between sleep and obesity are poorly understood. Sleep duration can alter brain functions involved in the control of appetite, which can in turn lead to overeating in an obesogenic environment [5]. Meanwhile, the associations between sleep duration and appetite are likely to be mediated by multiple factors, including a change in the levels of the appetite-related hormone leptin evoked by sleep deprived [6]. Leptin, a hormone secreted by white adipocytes, acts on specific receptors in the hypothalamus, a key tissue in satiety regulation and day-night circadian, and thus has been recently proposed as one possible mechanistic link between decreased habitual sleep duration and increased risk of obesity in both adults [7] and children [8-12]. In our recent cohort study of children from China with risk for metabolic syndrome (MS) [13], we also found that short sleep duration was associated with both increased obesity risk and leptin concentrations. However, the studies concerning the association between leptin levels and sleep duration are still inconsistent [14] and the underlying mechanisms still need to be elucidated.

A number of genome-wide association studies have revealed compelling genetic signals influencing obesity risk both in adults and children [15]. Recently, we replicated the genetic associations of established East Asian adult body mass index (BMI) loci [16-18] with childhood obesity, and found that six previously identified brain-expressed obesity-related loci, namely FTO-rs1558902, MC4R-rs2331841, MAP2K5rs4776970, GNPDA2-rs16858082, PCSK1-rs261967, and $B D N F$-rs2030323, exhibited significant association with both obesity risk and increasing leptin concentrations in children [19]. Importantly, previous studies have found that sleep duration could modify the effect of the FTO locus on both pediatric and adult BMI [20, 21]. Notably, other loci such as $M C 4 R, P C S K 1$, and BDNF, are well known to be associated with severe early-onset obesity and harbor genes involved in the regulation of leptin-melanocortin pathways in the hypothalamus [15], thus are thought to affect body weight largely through impacting appetite. As such, these findings implicate both common variants and sleep duration as exerting an effect on weight through leptin-related appetitive pathways, thus raising the possibility that there are gene-sleep interactions that function via a central regulatory mechanism, such as the leptin pathway therefore conferring early risk of obesity. However, evidence from longitudinal studies would be useful to address these issues but such datasets remain scarce.

This study leverages a large sample from the Beijing Child and Adolescent Metabolic Syndrome (BCAMS) study[22] in order to test the hypothesis that sleep duration in childhood not only modifies the genetic predisposition to childhood obesity, but also has long term impact on obesity risk, as assessed at a ten-year follow-up, particularly for loci operating via the leptin pathway. Our study provides novel insight into the links between gene-environment interaction and obesity that could be targeted to reduce risk in early life.

\section{Subjects and methods}

\section{Population}

BCAMS was designed as an ongoing follow-up study of obesity and related metabolic abnormalities among a representative sample of school-aged children in Beijing, which has been described in detail elsewhere (Supplementary Fig. 1) [22, 23] and was registered at www.clinicaltria 1s.gov (NCT03421444). Briefly, the BCAMS study recruited 19,593 school children (6-18 years old) via stratified randomized sampling from four urban and three rural districts within the Beijing area between April and October 2004. Based on initial finger capillary blood tests, 4500 participants at risk of MS were identified as having one or more of the following disorders: being overweight, high blood pressures, increased total cholesterol $\geq 5.2(\mathrm{mmol} / \mathrm{L})$, triglyceride $\geq 1.7(\mathrm{mmol} / \mathrm{L})$, or fasting glucose $\geq 5.6(\mathrm{mmol} / \mathrm{L})$. Next, all children at risk of MS $(n=4500)$, together with a parallel reference sample of 1095 school children, were invited to participate in a medical examination including venipuncture-based blood sample tests. In total, 3514 participants agreed to complete further medical examination. Among them, 3211 participants (1161 without any MS component based on further examination) who had completed the baseline full examination of leptin level, genotype, and lifestyles questionnaire including sleep time were included in this study [13]. A follow-up study was conducted after 10 years of initial investigation; participants were recruited consecutively through various modalities in a center at the Beijing Chaoyang Hospital. A total of 848 participants who completed anthropometric measurements at follow-up, including 559 who completed in-depth clinical examination $[23,24]$, were included in the longitudinal analysis. Thus, our analysis consisted of two parts: a cross-sectional analysis using baseline data $(n=3211)$ and a prospective analysis based on follow-up data $(n=848)$, which is described in detail in Supplementary Fig. 1. Informed consent from participants and/or parents/guardians was obtained prior to entry 
into the study. The BCAMS study was approved by the Ethics Committee at the Capital Institute of Pediatrics in Beijing. All the phases of the study complied with the Ethical Principles for Medical Research Involving Human Subjects expressed in the Declaration of Helsinki.

\section{Phenotyping}

The subjects' height, weight, waist circumference, and percent body fat (FAT \%) were measured according to our standard protocol $[19,22,23]$. Baseline leptin was measured by enzyme-linked immunosorbent assay with the intra- and inter-assay coefficient of variations $<7.4 \%$ and $<9.3 \%$, respectively [25]. All samples were tested in duplicate and blinded. BMI was calculated as weight/height [2]. According to the Working Group for Obesity in China, age- and sex-specific BMI percentiles were used to define overweight (85th) and obesity (95th) for children and adolescents ( $\leq 18$ years) [26]. For adults ( $>18$ years), obesity was defined as BMI $>28 \mathrm{~kg} / \mathrm{m}^{2}$ and overweight as BMI $24.0-28.0 \mathrm{~kg} / \mathrm{m}$ [27]. The diagnosis of MS in children and adults has been described in detail elsewhere [22, 23].

\section{Single nucleotide polymorphism (SNP) selection and genotyping}

Genomic DNA was isolated from peripheral white blood cells using the QIAamp DNA Blood Midi Kits (Qiagen). Twelve SNPs were selected from GWAS reports of obesity in East Asian ancestry populations [16-18], with particular focus on the loci harboring brain-expressed genes, such as FTOrs1558902, MC4R-rs2331841, BDNF-rs2030323, MAP2K5rs4776970, GNPDA2-rs16858082, and PCSK1-rs261967 (Supplementary Table 1). All SNPs were genotyped on the Sequenom Mass Array iPLEX genotyping platform [28]. Repeated control samples were present in each genotyping plate, yielding a concordance rate of $100 \%$.

\section{Sleep duration}

Self-reported sleep duration at baseline was obtained from all participants ( $>12$ years old) and/or their parents or guardians ( $\leq 12$ years old) and was derived from the following question: "How many hours did you sleep on an average day over the past 7 days?" The response ranged from 4.5 to $13 \mathrm{~h}[13]$. Sleep duration was categorized into four groups as follows: $\leq 7 \mathrm{~h} /$ day, $8 \mathrm{~h} /$ day, $9 \mathrm{~h} /$ day, and $\geq 10 \mathrm{~h} /$ day, or two groups based on the median of sleep time.

\section{Covariates}

At baseline, pubertal development by Tanner stage of breast development in girls and testicular volume in boys was assessed by a pediatrician of the same sex as the child. Lifestyle information was collected by questionnaire. Physical activity was expressed as low ( $<3$ times/week) or moderate-to-vigorous ( $\geq 3$ times/week), meaning that each individual spent $\geq 30 \mathrm{~m} /$ activity for extracurricular physical activities (cycling, hiking, batting, running, and swimming etc.). Dietary information included ten items (breakfast, bean, seafood, milk, vegetables, fruits, red meat, sweet soft drinks, snacks, and consumption of western-fast food). The response options were represented as $1-5$ depending on the degree of dietary status from "seldom or never" to "everyday" and diet scores were summed for each subject. A higher score indicated better dietary quality, and vice versa [28].

\section{Statistical analysis}

Analyses were performed using the Statistical Package for Social Sciences (SPSS) 20.0 for Windows. Hardy-Weinberg equilibrium was assessed using the Chi-squared test in the total sample. In this study, all SNPs passed Hardy-Weinberg equilibrium. Leptin was natural logarithmically (ln) transformed before analysis due to skewed distribution. By applying Bonferroni correction, a $P$-value below 0.004 $(0.05 / 12$ SNPs) was considered significant, while a $P$-value between 0.05 and 0.004 was considered nominally significant. A score of 0,1 , or 2 was assigned to genotypes of the BMI-associated SNPs according to the number of risk alleles in an additive model. We calculated a set of genetic predisposition scores (GPS) as follow: the GPS $_{\text {all }}$ for all 12 selected SNPs, the GPS leptin $_{\text {for six leptin-related SNPs }}$ (Supplementary Table 1) [19]. The GPSs were calculated as the sum of risk genotypes on the basis of single SNP analyses with the additive model.

Logistic or linear regression analyses were applied to test the associations between the GPSs, sleep duration, and obesity measurement. GPS $\times$ sleep duration interaction analysis for BMI was performed using a linear regression model with SNPs coded in an additive model, including potential confounders including age, sex, residence, puberty, exercise and diet score, and sleep duration coded as a dichotomous/continuous variable. SNP $\times$ sleep duration interaction analysis for obesity and overweight was performed using logistic regression with adjustment for the same covariates. When interactions were statistically significant, stratified analyses were undertaken to observer effect modification. Because the standard definitions for sleep duration norms specific to metabolic health in Chinese population have not yet been defined. According to the 2015 US National Sleep Foundation's recommendations [29], there are minor differences in the recommended sleep duration for youth: 9-11 h/day is recommended for children of school-age (6-13 years), and 8-10 h/day is recommended 
for teenagers (14-17 years). So regardless of age group, sleeping $\leq 7 \mathrm{~h}$ /day in children is not recommended and are known as short sleepers. Therefore, we classified the participants into four groups, beginning with $\leq 7 \mathrm{~h} / \mathrm{day}$, in order to observe the influence of every additional hour of sleep on the genetic susceptibility to childhood obesity. In addition, to exam the long term effect of sleep duration based on 10-year follow-up, we also classified the participants into two groups based on the median of sleep time $(<9 \mathrm{~h} /$ day and $\geq 9 \mathrm{~h}$ /day) to increase the power of the analysis.

Mediation analysis conducted on the whole population was performed using PROCESS Procedure for SPSS Release 2.12 [30] for baseline and 10-year follow-up BMI, respectively, adjusting for baseline age, sex, Tanner stage, residence, and behavioral factors. Our previous studies have confirmed that leptin plays a mediating role, not only in the association between sleep duration and baseline BMI [13], but also in the association between genetic predisposition and baseline BMI [19]. In this study, first we extended to analyze whether leptin plays a role in mediating the prospective association between the polygene's effect and risk of obesity at 10-year follow-up by using the template Model 4 (Fig. 1) [30]. Second, we tested whether sleep duration modifies the association between the respective polygene score and both baseline and follow-up BMI through leptin levels by using Model 8 [30]. As depicted in Fig. 2, the proposed moderator variable was baseline sleep duration, the model was used to test for an effect of the GPS-sleep interaction on baseline and follow-up BMI; the proposed mediator variable was baseline leptin levels, where this model was used to test the paths from GPS, sleep duration, and GPSsleep interaction, respectively, to Ln-leptin, and from the Lnleptin to change in BMI at both baseline and follow-up.

\section{Results}

\section{Cohort characteristics}

Baseline characteristics of the participants according to baseline and follow-up weight categories are listed in Table 1. The study was conducted in 3211 baseline and 848 followup unrelated individuals recruited through the BCAMS study. For baseline weight categories, $50.0 \%$ of the participants were male, and the mean age was $12.4 \pm 3.1$ years, and the average sleep duration was $8.5 \mathrm{~h}$ (median $9.0 \mathrm{~h}$ ). Compared with the participants presenting as normal weight and overweight, a higher proportion of the children with obesity were prepubertal and male. The obesity phenotype was associated with younger age, and less frequent physical activity (all $P<0.05$ ). Moreover, obesity-related anthropometric traits (i.e., BMI, waist circumference, and FAT \%) and leptin were increased gradually from normal weight to overweight and obese groups (all $P<0.001$ ). As expected, the significant differences in the baseline adiposity traits remained (all $P<0.001$ ) when the participants were classified by 10 -year follow-up body weight. Moreover, among the 848 participants who returned for follow-up, $3 \%$ of the children presenting as normal weight and $12 \%$ as overweight developed obesity at 10-year follow-up, while over $85 \%$ of the children with obesity at baseline remained obese at 10-year follow-up, suggesting a well-established obesity trajectory over time from childhood to adulthood.

\section{Sleep duration modifies the association between genetic predisposition and adiposity, both at baseline and at follow-up}

Our previous cross-sectional study confirmed similar studywide significant association with the baseline BMI and obesity for the polygene score consisting of all the 12 adult BMI loci $\left(\mathrm{GPS}_{\mathrm{all}}\right)$ and the $\mathrm{GPS}_{\text {leptin }}$ of 6 leptin-related loci [19]. In this study, we also confirmed study-wide significant association with risk of obesity and overweight at 10-year follow-up for either $\mathrm{GPS}_{\text {all }}(\mathrm{OR}$ [95\%CI]: 1.138 [1.066, 1.214]; $P=9.3 \times 10^{-5}$ ) or $\operatorname{GPS}_{\text {leptin }}$ (1.183 [1.079, 1.296]; $P=3.2 \times 10^{-4}$ ) (Supplementary Table 1 ); however, no association for GPS after excluding the six leptin-related SNPs was observed, thus further polygene analyses were restricted to $\mathrm{GPS}_{\text {leptin. }}$.

Next, we tested for association of the six leptin-related variants and GPS $_{\text {leptin }}$ with continuous sleep duration and dichotomized short or long sleep duration, adjusting for age, sex, and other lifestyle factors. No individual variant or GPS $_{\text {leptin }}$ yielded significant association with sleep duration (Supplementary Table 2).

We then examined whether sleep duration modifies the effect of key variants on the risk of obesity by testing for interaction between baseline sleep duration and GPS $_{\text {leptin }}$ in influencing BMI/obesity and overweight at both baseline $(n=3211)$ and 10-year follow-up $(n=848)$. Significant $\mathrm{GPS}_{\text {leptin }} \times$ sleep duration interaction was observed on baseline BMI/obesity and overweight $(P$ interaction $=0.008$ for BMI, $P$ interaction $=0.002$ for obesity and overweight). We further conducted stratified analyses by categories of sleep duration. As shown in Table 2, for participants sleeping $\leq 7 \mathrm{~h}$ /day at baseline, after adjusting for age, sex, and other lifestyle factors, GPS $_{\text {leptin }}$ was associated with significantly higher baseline BMI ( $\beta$ [95 CI\%]: 0.723[0.444-1.001] $\mathrm{kg} / \mathrm{m}^{2}, P=5.2 \times 10^{-7}$ ) and obesity and overweight risk (OR [95\%CI]: 1.328 [1.164-1.516], $P=2.6 \times 10^{-5}$ ); for participants sleeping $8 \mathrm{~h} /$ day $\left(P=6.5 \times 10^{-4}\right)$ or $9 \mathrm{~h} /$ day $(P=0.003)$, the positive associations between $\mathrm{GPS}_{\text {leptin }}$ and $\mathrm{BMI} /$ obesity and overweight were attenuated to a degree; however, in participants whose sleep duration $\geq 10 \mathrm{~h} /$ day, the associations were no longer significant $(P>0.05)$. In 
Fig. 1 A visual schematic of the path model used to test the mediation effect of leptin on the association of GPS leptin $_{\text {with }}$ baseline and follow-up BMI. Flow diagram showing that the mediation effect using Ln-leptin was $63.7 \%$ for baseline BMI and $70.6 \%$ for follow-up BMI. $\beta$ for linear regression in the mediation models was adjusting for age, sex, residence, pubertal stages, diet score, and activity. $* P \leq$ $0.05, * * P \leq 0.01, * * * P \leq 0.001$. BMI body mass index, GPS genetic predisposition scores
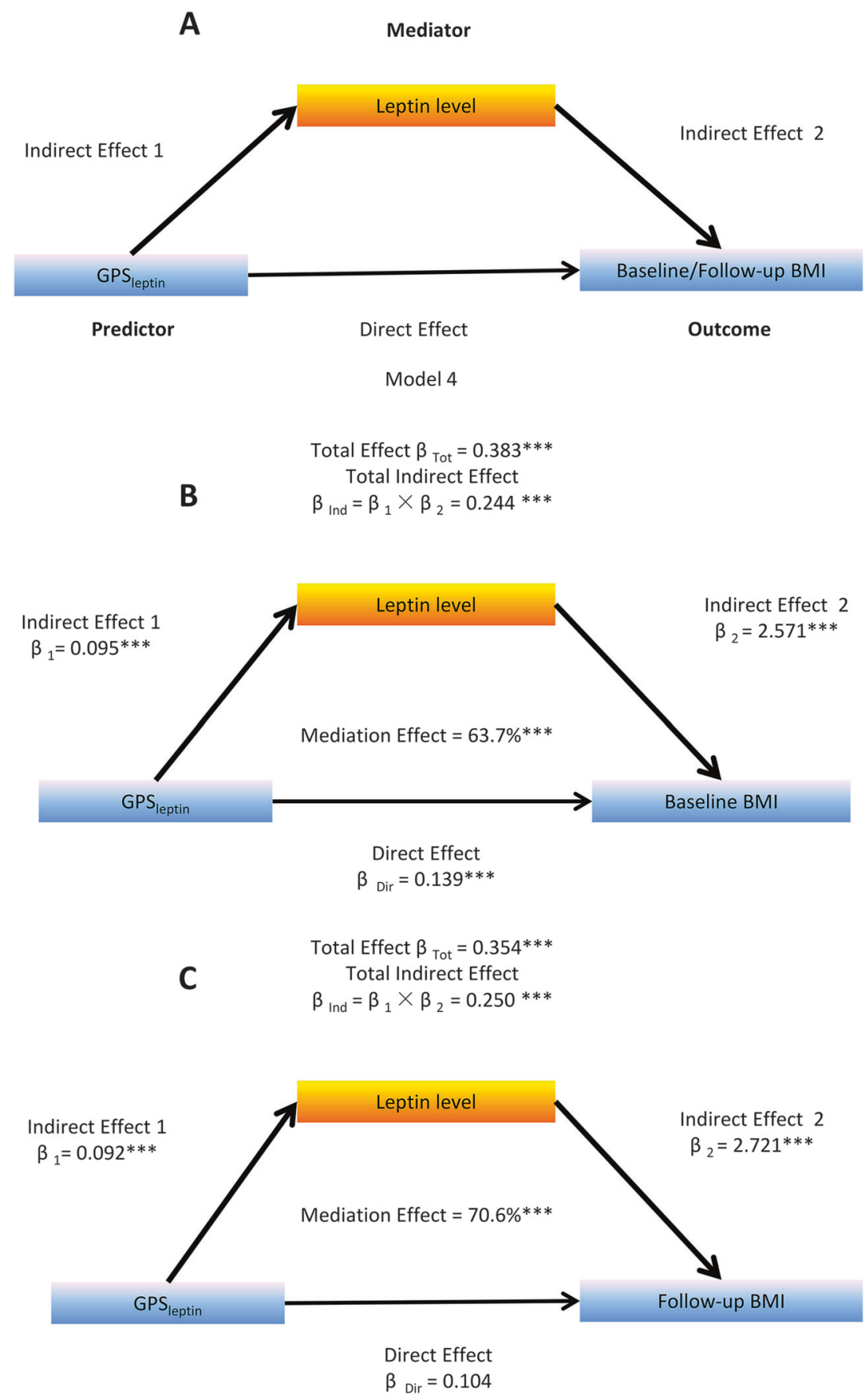

addition, the results were consistent when analyses were stratified by sex. At the follow-up phase, to increase the study power, we combined participants into two groups according to the median of baseline sleep duration ( $<9 \mathrm{~h} /$ day and $\geq 9 \mathrm{~h} /$ day). After adjusting for multiple confounders, GPS $_{\text {leptin }}$ was significantly associated with obesity and overweight risk (OR [95\%CI]: 1.300 [1.093-1.546], $P=$ 0.003 ) in participants sleeping $<9 \mathrm{~h} / \mathrm{day}$, but the association disappeared in participants whose sleep duration $\geq 9 \mathrm{~h} /$ day $(P>0.05)$. Moreover, the sleep duration could modify the effects of the $F T O$-rs 1558902 risk alleles $(P$ for interaction $=0.018)$ on BMI at baseline, while not evident in other leptin-related SNPs (Supplementary Table 3). In addition, we analyzed whether sleep duration could modify the effect of $\mathrm{GPS}_{\text {all }}$ on BMI/obesity and overweight and found the interaction was very similar to GPS $S_{\text {leptin. }}$. 
Fig. 2 A visual schematic of the path model used to test the moderation effect of sleep duration and the mediation effect of leptin on the association of GPS $_{\text {leptin }}$ with baseline and follow-up BMI. The proposed moderator variable was baseline sleep duration, the model was used to test for an effect of the GPS-sleep interaction on baseline and follow-up BMI; the proposed mediator variable was baseline leptin levels, where this model was used to test the paths from GPS, sleep duration, and GPSsleep interaction respectively to Ln-leptin, and from the Ln-leptin to change in BMI at both baseline and follow-up. $\beta$ for linear regression in the mediation models was adjusting for age, sex, residence, pubertal stages, diet score, and activity. ${ }^{*} P \leq$ $0.05, * * P \leq 0.01, * * * P \leq 0.001$ BMI body mass index, GPS genetic predisposition scores
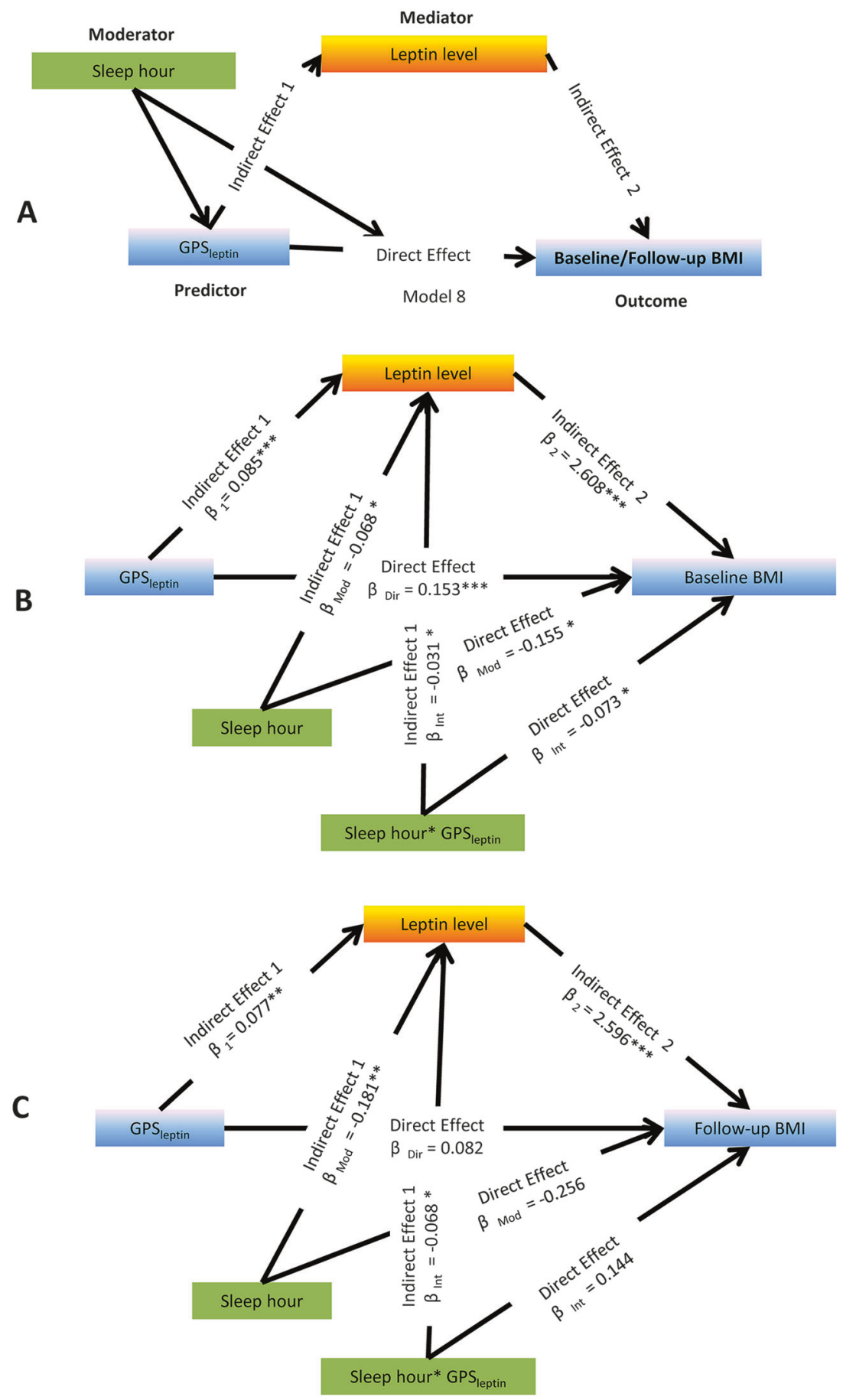

\section{Leptin mediated the association between genetic predisposition and adiposity}

To exam whether leptin mediates the effect of polygenic risk on obesity from childhood to young adulthood, we tested for mediation effect of baseline leptin on GPS $_{\text {leptin-BMI associa- }}$ tions adjusting for baseline age, sex, residence, pubertal stages, and other lifestyle factors. As listed in Fig. 1, the total effect of
$\mathrm{GPS}_{\text {leptin }}$ on baseline $\left(\beta_{\text {Total }}=0.383, P<0.001\right)$ and 10 -year follow-up BMI $\left(\beta_{\text {Total }}=0.354, P<0.001\right)$ were estimated without leptin in the models. The total indirect effect via leptin defined as the product of indirect effect $1\left(\beta_{1}\right)$ and indirect effect $2\left(\beta_{2}\right)$ was significant at both baseline $\left(\beta_{\text {Indirect }}=0.244\right.$, $P<0.001)$ and follow-up BMI $(\beta$ Indirect $=0.250, P<0.001)$. The net mediation effects of leptin on baseline and follow-up BMI were $63.7 \%$ and $70.6 \%$, respectively. 


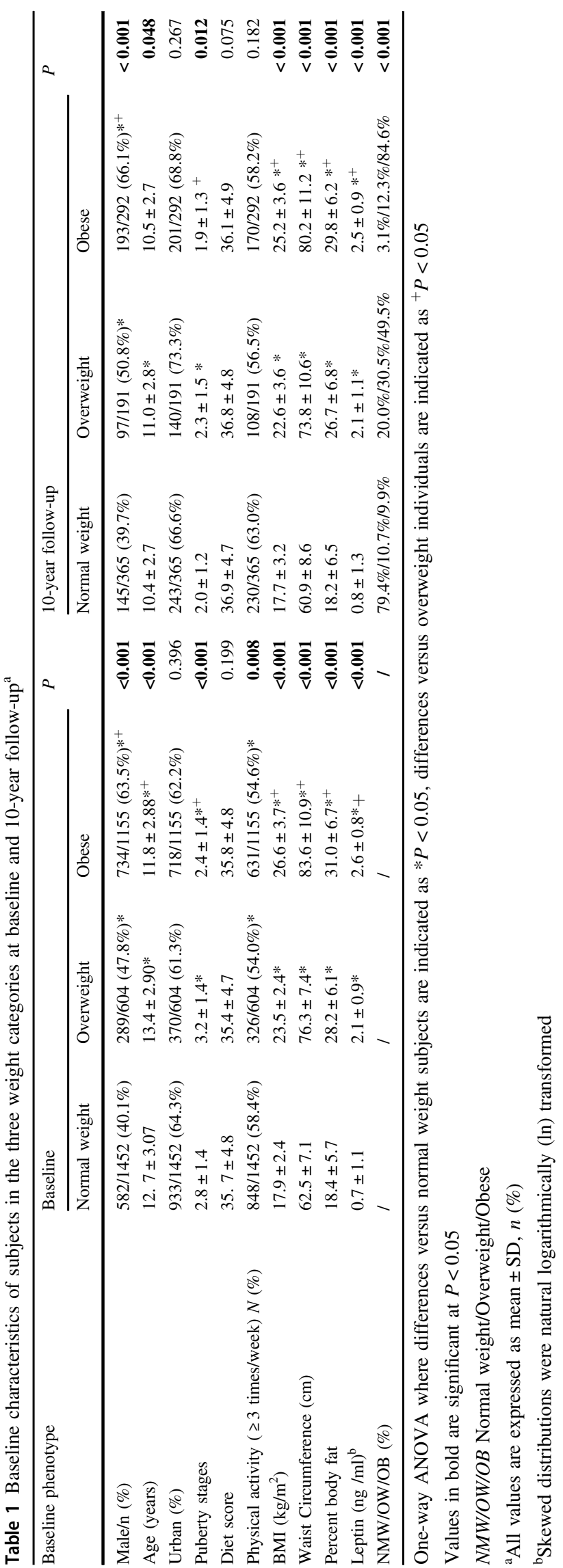

\section{Moderation of sleep duration and mediation of leptin on the association between genetic predisposition and adiposity}

We sought to test for both the moderation of sleep duration and the mediation of leptin on polygenic risk influencing BMI with a long-term effect (visual schematic of the path model shown in Fig. 2). At baseline, we observed nominally significant interactions between $\mathrm{GPS}_{\text {leptin }}$ and sleep duration for BMI and leptin concentrations, with a $0.073 \mathrm{~kg} / \mathrm{m}^{2}$ lower BMI and $0.031 \mathrm{ng} / \mathrm{ml}$ lower Ln-leptin with each additional sleeping hour per risk allele, and the total indirect mediation effect of baseline leptin $\left(\beta=-0.081 \mathrm{~kg} / \mathrm{m}^{2}, P<\right.$ 0.05 ) on the $\mathrm{GPS}_{\text {leptin-childhood BMI association was }}$ $52.6 \%$. Meanwhile, longer sleep duration weakened the effect of $\mathrm{GPS}_{\text {leptin }}$ on leptin at 10-year follow-up, with a $0.068 \mathrm{ng} / \mathrm{ml}$ lower Ln-leptin with each additional sleeping hour per risk allele, while there was a significant indirect

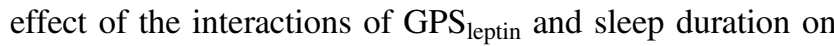
10-year follow-up BMI $(\beta=-0.177, P<0.05)$; however, the mediated effect of leptin was not in the same direction as the direct effect, thus the proportion mediated was undefined for follow-up BMI. Table 3 summarizes the conditional direct and indirect effects of GPS $\mathrm{Geptin}_{\text {lin }}$ on baseline and 10 -year follow-up BMI at the mean values $\pm 1 \mathrm{SD}$ of the baseline sleep duration. The moderation effect of sleep duration yielded a significant direct effect of $0.153 \mathrm{~kg} / \mathrm{m}^{2}$ and indirect effect of $0.223 \mathrm{~kg} / \mathrm{m}^{2}$ (all $P<0.05$ ) on the baseline BMI, while only appeared a significant indirect effect of $0.199 \mathrm{~kg} / \mathrm{m}^{2}(P<0.05)$ on the follow-up BMI. Meanwhile, both the direct and indirect effects were attenuated with the increase in sleep duration, with the finding indicating that sleep duration modified the polygenic obesity risk mainly via the leptin pathway to impact 10 -year follow-up BMI.

\section{Discussion}

In this cohort study of youth from China, we report evidence for interaction between sleep duration and genetic factors in the modulation of body weight at baseline and at 10-year follow-up. The most notable finding is that the interaction effect of the polygene risk score and baseline sleep duration on BMI was significantly mediated by elevated leptin concentration. Therefore, our results indicate that adequate sleep time in childhood appears to reverse the adverse effects of BMI-related loci on obesity from childhood to adulthood, particularly through the leptin pathway.

Our results confirm and extend the association between self-reported short sleep duration and risk of obesity observed in previous studies, both in adult [31-33] and in children $[34,35]$. Meanwhile, the central nervous system 


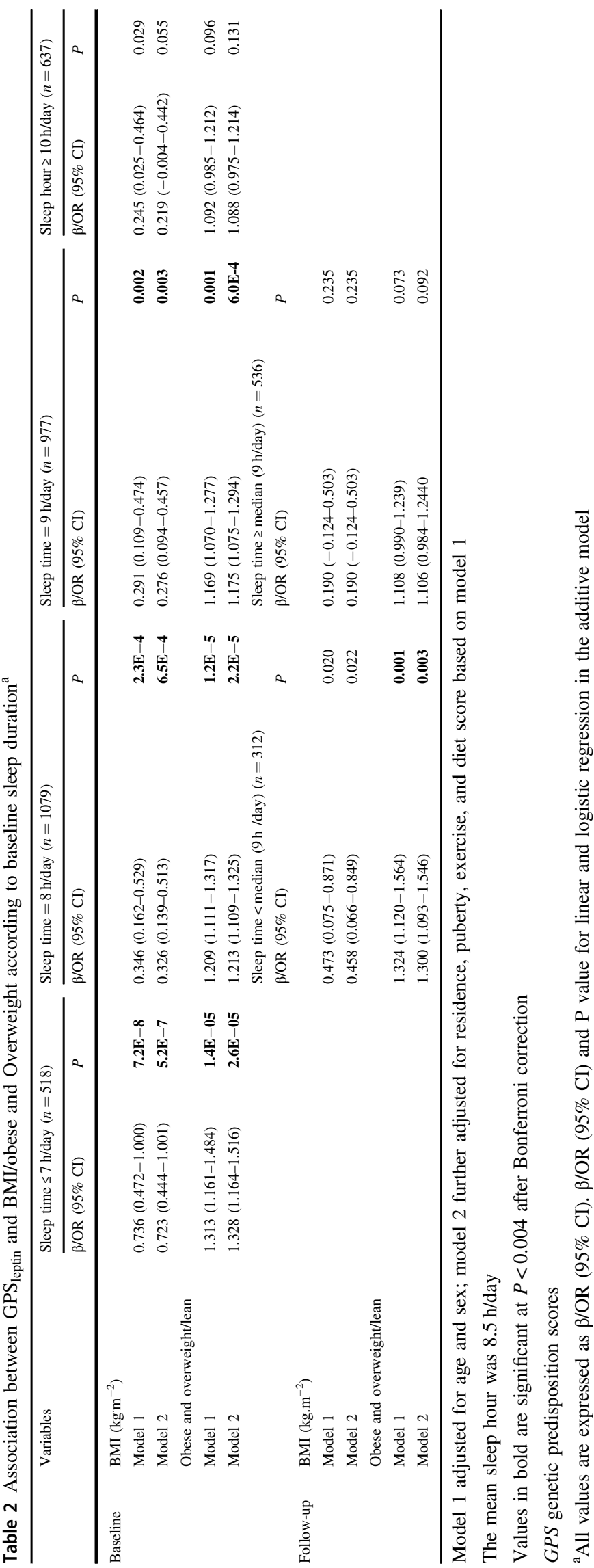

(CNS), especially the hypothalamus is currently considered to be primarily responsible for controlling appetite and body weight. Sleep disturbances can influence brain functions involved in appetite and energy balance regulation, which are related to obesity, and higher risk of cardio-metabolic disorders [31, 33, 36]. Notably, the brain-expressed genes located at key BMIrelated loci, such as FTO, MC4R, MAP2K5, GNPDA2, $P C S K 1$, and $B D N F$, may yield many connections with sleep duration. As far as we are aware, the only previous study reporting an $F T O$-sleep duration association found evidence that TT homozygotes (but not $\mathrm{A}^{*}$ carriers) for the FTO SNP rs9939609 (in high LD with the SNP we studied, rs1558902, $r_{\mathrm{CEU}}^{2}=0.922$ ) was nominally associated with decreasing sleep duration in Caucasian children aged 7 [37]. A role for $B D N F$ in sleep homeostasis has also been proposed [38-40]. BDNF mRNA levels are higher after wakefulness and BDNF levels were positively correlated with the duration of the following sleep period [38, 39]. However, no data is available regarding the relationships between the other four genes (MC4R, MAP2K5, GNPDA2, and PCSK1) and sleep duration. In this study, we observed no significant associations between sleep duration and the six leptin-related SNPs and GPS $_{\text {leptin }}$, indicating that sleep duration may act as a modifier rather than a mediator in the association between genetic risk and pediatric BMI.

As previous authors have argued, shorter sleep duration (sleep $<7 \mathrm{~h} /$ day) increases the expression of genetic risks for high BMI in adults from the US (mean 36.6 years) [20], implying that the genetic effects on BMI differ depending on sleep. Recently, Young et al. reported that squared deviations from mean sleep duration $(7.18 \mathrm{~h})$ are associated with an enhanced effect of FTO-rs1421085 on adult (mean 56.8 years) BMI [21]. In this study, first, we found that short sleep duration in childhood enhanced the effect of FTO-rs1558902 on pediatric BMI but not with BMI of grown-ups (likely due to less study power in follow-up population). Second, sleep duration significantly mediated the association between adiposity and polygenic obesity risk score $\left(\mathrm{GPS}_{\text {leptin }}\right)$ that excluded FTO both in childhood and adulthood $(P<0.05)$, thus, the associations observed in our study were not explained entirely by variant residing in FTO (data not shown). Our finding supports the hypothesis that one variant contribute only to a small degree, while additive polygenic SNP models have a more significant effect on common obesity.

Notably, we found that childhood sleep duration significantly modulated the association between polygenic obesity risk score $\left(\mathrm{GPS}_{\text {leptin }}\right)$ and pediatric and the 10-year follow-up BMI. This suggests that inadequate sleep time in early life has long-term adverse impact on 
Table 3 Direct and indirect effects of GPS $_{\text {leptin }}$ on baseline and 10-year follow-up BMI at different values of the sleep duration at baseline ${ }^{\mathrm{a}}$

\begin{tabular}{|c|c|c|c|c|c|c|c|c|c|}
\hline \multicolumn{6}{|l|}{ Baseline BMI } & \multicolumn{4}{|c|}{ Follow-up BMI } \\
\hline \multicolumn{10}{|c|}{ Conditional direct effects of GPS leptin on baseline and 10-year follow-up BMI at values of the sleep duration: } \\
\hline Sleep duration & Effect & $S E$ & $95 \% C I$ & $P$ & Sleep duration & Effect & $S E$ & $95 \% C I$ & $P$ \\
\hline$-1 \mathrm{SD}(1.0 \mathrm{~h})$ & 0.224 & $\mathbf{0 . 0 5 0}$ & $0.126-0.322$ & $7.5 \mathrm{E}-6$ & $-1 \mathrm{SD}(1.0 \mathrm{~h})$ & -0.040 & 0.142 & $-0.319--0.239$ & 0.778 \\
\hline Mean (8.5 h) & 0.153 & 0.036 & $0.083-0.223$ & $1.8 E-5$ & Mean $(8.5 \mathrm{~h})$ & 0.082 & 0.101 & $-0.116-0.280$ & 0.416 \\
\hline$+1 \mathrm{SD}(1.0 \mathrm{~h})$ & 0.082 & 0.050 & $-0.0162-0.180$ & 0.102 & $+1 \mathrm{SD}(1.0 \mathrm{~h})$ & 0.204 & 0.143 & $-0.077-0.485$ & 0.154 \\
\hline \multicolumn{10}{|c|}{ Conditional indirect effects of GPS leptin on baseline and 10-year follow-up BMI at values of the sleep duration: through Ln-leptin } \\
\hline Sleep duration & Effect & $S E$ & $95 \% C I$ & & Sleep duration & Effect & $S E$ & $95 \% C I$ & \\
\hline$-1 \mathrm{SD}(1.0 \mathrm{~h})$ & 0.302 & 0.049 & $0.203-0.395$ & $<0.05$ & $-1 \mathrm{SD}(1.0 \mathrm{~h})$ & 0.348 & 0.096 & $0.165-0.544$ & $<0.05$ \\
\hline Mean (8.5 h) & 0.223 & 0.038 & $0.148-0.301$ & $<0.05$ & Mean $(8.5 \mathrm{~h})$ & 0.199 & 0.075 & $0.051-0.348$ & $<0.05$ \\
\hline$+1 \mathrm{SD}(1.0 \mathrm{~h})$ & 0.143 & 0.057 & $0.035-0.257$ & $<0.05$ & $+1 \mathrm{SD}(1.0 \mathrm{~h})$ & 0.049 & 0.112 & $-0.174-0.267$ & $>0.05$ \\
\hline
\end{tabular}

$\beta(95 \% \mathrm{CI})$ and $P$ value for linear regression in the mediation models

$P$ value was adjusting for age, sex, residence, pubertal stages, diet score, and activity at baseline

Values for moderators i.e., sleep durations are the mean and \pm 1 SD from mean

Values in bold are significant at $P<0.05$

GPS genetic predisposition scores, $B M I$ body mass index

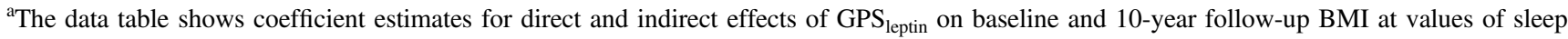
duration at baseline

genetically susceptible individuals through the development of obesity.

However, the mechanisms by which inadequate sleep increases the genetic risk effect on obesity pathogenesis are poorly understood. Currently, adipose tissue has been recognized as a dynamic endocrine organ, producing a range of biologically active substances, collectively called "adipokines", which regulate energy homeostasis, inflammation, insulin resistance, and cardiovascular function [41]. Among the adipokines, leptin is the most thoroughly examined in the literature, and is recognized as a fundamental regulator of energy homeostasis, acting through the binding of its functional long isoform leptin receptor in the CNS to control feeding behavior and energy expenditure, and modulating glucose/lipid metabolism in peripheral tissues [42]. However, there is an ongoing debate on the association between sleep duration and leptin levels [14]. Previous studies have revealed that leptin levels are variable with short sleep duration, either increased $[9,11,14]$ or decreased $[8,14]$. In our recent cohort study of children with risk for MS [13], we have reported that sleep duration was negatively associated with circulating leptin levels even after adjustment for BMI. Given our cohort consists of a high proportion of participants with overweight and obesity (54.8\%), among whom hyperleptinemia or leptin resistance were relatively common, our finding can be interpreted as chronic lack of sleep reducing leptin sensitivity; thus, an elevation in circulating leptin may reflect a secondary increase in an attempt to overcome this leptin resistance. In addition, we also found that leptin could mediate the association between sleep duration and cardiometabolic risk factors, and accounted for $81.6 \%$ of the total effect of sleep duration on BMI [13]. Based on our current analysis, it is likely that the childhood sleeping time acts as a long term modifier on the effect of polygenic risk for obesity through the leptin pathway.

Among the six leptin-associated CNS-highly-expressed loci, three genes (MC4R, BDNF, and PCSK1) are known to be involved in the hypothalamic leptin-melanocortin pathway $[15,43,44]$. FTO is the first GWAS-identified obesitysusceptibility locus and shows the strongest association with BMI in our pediatric cohort [19]. Unlike the abovenoted three genes with possible function through hypothalamic leptin-melanocortin pathway, FTO modulates leptin receptor localization within neurons to control food intake and adiposity [45]. However, there is much debate on which is the actual effector gene(s) at this locus. Furthermore, $M A P 2 K 5$ and GNPDA2 are also highly-expressed in CNS and positive correlated with leptin [19]. Given the fact that the genes at these six loci are parts or have well-established connections with leptin in literature, it is not surprising that we found the modifying effect of sleep duration partly via leptin.

On the other hand, we analyzed the modification of sleep duration on the effect of GPS all (all the 12 selected SNPs)

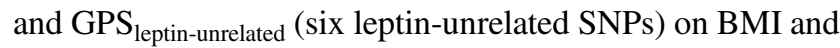
leptin. However, we observed no significant interactions between GPS all $_{\text {, }}$, GPS $_{\text {leptin-unrelated, and sleep duration on }}$ baseline concentrations of leptin (data not shown). The results further confirmed our hypothesis that sleep duration

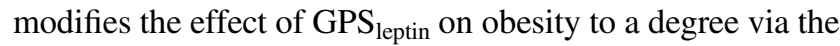
leptin pathway. 
This study has a number of strengths. This is the first longitudinal study to quantify the modification of sleep duration on polygenic obesity risk score and explore the possible mechanisms for their impact on BMI, providing novel intervention targets in early life. In addition, analyzing a large, well-characterized cohort of participants with a range of covariates makes our results more robust by adjusting for many potential confounders known to be associated with obesity. Nevertheless, there are several limitations that should be noted. First, mainly due to great migration to other parts of the country or to other countries as those school-aged children growing up with the rapid development of society and economy in Beijing, the number of participants returning to follow-up after 10 years was relatively small compared with our original population at baseline. However, this longitudinal cohort enables polygenic obesity risk assessment, providing sufficient power (83\%) to detect a significant causal association with our sample size of 848 participants. Second, we conducted a large longitudinal cohort study for the possible sleep-gene interaction for obesity risk, our results are considered as a credible reference for the children at risk of MS, however, it is not clear whether these results are further generalizable to all children. Third, our findings were obtained from participants from China exclusively and therefore cannot be generalized to other ethnic populations without further replication. In addition, participants in our study have a large age span at baseline, especially have a small number at follow-ups; due to the relatively small sample size after age stratification, a larger sample size will be needed ultimately to explore the effects of sleep time on the risk of future obesity in different childhood age stratification. Moreover, the habitual sleep duration is recorded through self-report questionnaires rather than by laboratory examination, and therefore, may influence the accuracy of the data. However, the advantage of using such approach is the cost-effectiveness in the context of large sample size. Previous studies, especially for those with large sample sizes, they also comparably leveraged habitual sleep time data via a questionnaire approach [8-10]. Finally, there is a scarcity of information on the multiple sleep dimensions, e.g., patterns and quality.

In summary, our longitudinal study supports the notion that increased sleep time during childhood has advantageous effects on protecting against a genetic predisposition to obesity, with leptin playing a key role in the process. Therefore, we recommend getting adequate sleep in childhood as an important intervention to prevent obesity, especially if there is a genetic predisposition to this trait. As these are among the only longitudinal data on the interaction between sleep duration and polygenic factors in the modulation of body weight, further prospective studies with larger sample size from diverse pediatric populations are warranted to further validate our findings.
Acknowledgements The authors thank Dr. Jie Mi, the professor of Capital Institute of Pediatrics in Beijing, and the other BCMAS study members and participants for their continuing participation in this research effort.

Funding This work was supported by the National Key Research program of China (2016YFC1304801); Key Program of Beijing Municipal Science and Technology Commission (D111100000611001, D111100000611002); Beijing Natural Science Foundation (7172169); Beijing Science and Technology Star Program (2004A027); Novo Nordisk Union Diabetes Research Talent Fund (2011A002); National Key Program of Clinical Science (WBYZ2011-873); the Non-profit Central Research Institute Fund of Chinese Academy of Medical Sciences (2017PT32020, 2018PT32001); and Jingxi Scientific Program of Beijing Chaoyang Hospital (JXPY201606). We gratefully thank all the participants in the "BCAMS study". Dr. Grant is funded by R01 HD056465 and the Daniel B. Burke Chair for Diabetes Research.

Author contributions JF analyzed the data and wrote the manuscript; SFG contributed to the design, data interpretation, and reviewed/edited the manuscript; YW, GL, LH, YL, LL, DF, and YW contributed to data collection; XX contributed to the data interpretation; MYL contributed to the data interpretation and reviewed the manuscript; SG was responsible for the follow-up study, and contributed to the design, data interpretation, and edited the manuscript; ML was responsible for the biomarker study of BCAMS, and contributed to the design, acquisition, and interpretation of the data, and revised the manuscript. All authors read and approved the final manuscript.

\section{Compliance with ethical standards}

Conflict of interests The authors declare that they have no conflict of interest.

Publisher's note: Springer Nature remains neutral with regard to jurisdictional claims in published maps and institutional affiliations.

Open Access This article is licensed under a Creative Commons Attribution 4.0 International License, which permits use, sharing, adaptation, distribution and reproduction in any medium or format, as long as you give appropriate credit to the original author(s) and the source, provide a link to the Creative Commons license, and indicate if changes were made. The images or other third party material in this article are included in the article's Creative Commons license, unless indicated otherwise in a credit line to the material. If material is not included in the article's Creative Commons license and your intended use is not permitted by statutory regulation or exceeds the permitted use, you will need to obtain permission directly from the copyright holder. To view a copy of this license, visit http://creativecommons. org/licenses/by/4.0/.

\section{References}

1. Afshin A, Forouzanfar MH, Reitsma MB, Sur P, Estep K, Lee A, et al. Health effects of overweight and obesity in 195 countries over 25 years. N Engl J Med. 2017;377:13-27.

2. Fatima Y, Doi SA, Mamun AA. Longitudinal impact of sleep on overweight and obesity in children and adolescents: a systematic review and bias-adjusted meta-analysis. Obes Rev. 2015;16:137-49.

3. Celis-Morales C, Lyall DM, Guo Y, Steell L, Llanas D, Ward J, et al. Sleep characteristics modify the association of genetic predisposition with obesity and anthropometric measurements in 119,679 UK Biobank participants. Am J Clin Nutr. 2017;105:980-90. 
4. Cappuccio FP, Taggart FM, Kandala NB, Currie A, Peile E, Stranges S, et al. Meta-analysis of short sleep duration and obesity in children and adults. Sleep. 2008;31:619-26.

5. St-Onge MP, Shechter A. Sleep disturbances, body fat distribution, food intake and/or energy expenditure: pathophysiological aspects. Horm Mol Biol Clin Invest. 2014;17:29-37.

6. Hayes AL, Xu F, Babineau D, Patel SR. Sleep duration and circulating adipokine levels. Sleep. 2011;34:147-52.

7. St-Onge MP. The role of sleep duration in the regulation of energy balance: effects on energy intakes and expenditure. J Clin Sleep Med. 2013;9:73-80.

8. Boeke CE, Storfer-Isser A, Redline S, Taveras EM. Childhood sleep duration and quality in relation to leptin concentration in two cohort studies. Sleep. 2014;37:613-20.

9. Hitze B, Bosy-Westphal A, Bielfeldt F, Settler U, PlachtaDanielzik S, Pfeuffer M, et al. Determinants and impact of sleep duration in children and adolescents: data of the Kiel obesity prevention study. Eur J Clin Nutr. 2009;63:739-46.

10. Martinez-Gomez D, Eisenmann JC, Gomez-Martinez S, Hill EE, Zapatera B, Veiga OL, et al. Sleep duration and emerging cardiometabolic risk markers in adolescents. The AFINOS study. Sleep Med. 2011;12:997-1002.

11. Hart CN, Carskadon MA, Considine RV, Fava JL, Lawton J, Raynor HA, et al. Changes in children's sleep duration on food intake, weight, and leptin. Pediatrics. 2013;132:e1473-80.

12. Kjeldsen JS, Hjorth MF, Andersen R, Michaelsen KF, Tetens I, Astrup A, et al. Short sleep duration and large variability in sleep duration are independently associated with dietary risk factors for obesity in Danish school children. Int J Obes. 2014;38:32-9.

13. Li L, Fu J, Yu XT, Li G, Xu L, Yin J, et al. Sleep duration and cardiometabolic risk among chinese school-aged children: do adipokines play a mediating role? Sleep. 2017;40:zsx042.

14. Felso R, Lohner S, Hollody K, Erhardt E, Molnar D. Relationship between sleep duration and childhood obesity: Systematic review including the potential underlying mechanisms. Nutr Metab Cardiovasc Dis. 2017;27:751-61.

15. Chesi A, Grant SF. The genetics of pediatric obesity. Trends Endocrinol Metab. 2015;26:711-21.

16. Wen W, Cho YS, Zheng W, Dorajoo R, Kato N, Qi L, et al. Metaanalysis identifies common variants associated with body mass index in east Asians. Nat Genet. 2012;44:307-11.

17. Wen W, Zheng W, Okada Y, Takeuchi F, Tabara Y, Hwang JY, et al. Meta-analysis of genome-wide association studies in East Asian-ancestry populations identifies four new loci for body mass index. Hum Mol Genet. 2014;23:5492-504.

18. Okada Y, Kubo M, Ohmiya H, Takahashi A, Kumasaka N, Hosono $\mathrm{N}$, et al. Common variants at CDKAL1 and KLF9 are associated with body mass index in east Asian populations. Nat Genet. 2012;44:302-6.

19. Fu J, Li G, Li L, Yin J, Cheng H, Han L, et al. The role of established East Asian obesity-related loci on pediatric leptin levels highlights a neuronal influence on body weight regulation in Chinese children and adolescents: the BCAMS study. Oncotarget. 2017;8:93593-607.

20. Watson NF, Harden KP, Buchwald D, Vitiello MV, Pack AI, Weigle DS, et al. Sleep duration and body mass index in twins: a gene-environment interaction. Sleep. 2012;35:597-603.

21. Young AI, Wauthier F, Donnelly P. Multiple novel gene-byenvironment interactions modify the effect of FTO variants on body mass index. Nat Commun. 2016;7:12724.

22. Li M, Fisette A, Zhao XY, Deng JY, Mi J, Cianflone K. Serum resistin correlates with central obesity but weakly with insulin resistance in Chinese children and adolescents. Int $\mathrm{J}$ Obes. 2009;33:424-39.
23. Fu J, Hou C, Li L, Feng D, Li G, Li M, et al. Vitamin D modifies the associations between circulating betatrophin and cardiometabolic risk factors among youths at risk for metabolic syndrome. Cardiovasc Diabetol. 2016;15:142.

24. Feng D, Zhang J, Fu J, Wu H, Wang Y, Li L, et al. Association between sleep duration and cardiac structure in youths at risk for metabolic syndrome. Sci Rep. 2016;6:39017.

25. Li M, Yin JH, Zhang K, Wu CY. A highly sensitive enzymelinked immunosorbent assay for measurement of leptin secretion in human adipocytes. Zhonghua Yi Xue Za Zhi. 2008;88:3293-7.

26. Group of China Obesity Task Force. Body mass index reference norm for screening overweight and obesity in Chinese children and adolescents. Zhonghua Liu Xing Bing Xue Za Zhi. 2004;25:97-102.

27. Chen $\mathrm{C}, \mathrm{Lu}$ FC. The guidelines for prevention and control of overweight and obesity in Chinese adults. Biomed Environ Sci. 2004;17(Suppl:):1-36.

28. Li L, Yin J, Cheng H, Wang Y, Gao S, Li M, et al. Identification of genetic and environmental factors predicting metabolically healthy obesity in children: data from the BCAMS study. J Clin Endocrinol Metab. 2016;101:1816-25.

29. Hirshkowitz M, Whiton K, Albert SM, Alessi C, Bruni O, DonCarlos L, et al. National sleep foundation's sleep time duration recommendations: methodology and results summary. Sleep Health. 2015;1:40-43.

30. Hayes AF. Partial, conditional, and moderated moderated mediation: quantification, inference, and interpretation. Commun Monogr. 2018;85:4-40.

31. Dean E, Bloom A, Cirillo M, Hong Q, Jawl B, Jukes J, et al. Association between habitual sleep duration and blood pressure and clinical implications: a systematic review. Blood Press. 2012;21:45-57.

32. Guo X, Zheng L, Wang J, Zhang X, Zhang X, Li J, et al. Epidemiological evidence for the link between sleep duration and high blood pressure: a systematic review and meta-analysis. Sleep Med. 2013;14:324-32.

33. Cappuccio FP, D'Elia L, Strazzullo P, Miller MA. Sleep duration and all-cause mortality: a systematic review and meta-analysis of prospective studies. Sleep. 2010;33:585-92.

34. Landhuis CE, Poulton R, Welch D, Hancox RJ. Childhood sleep time and long-term risk for obesity: a 32-year prospective birth cohort study. Pediatrics. 2008;122:955-60.

35. Touchette E, Petit D, Tremblay RE, Boivin M, Falissard B, Genolini C, et al. Associations between sleep duration patterns and overweight/obesity at age 6. Sleep. 2008;31:1507-14.

36. Dashti HS, Follis JL, Smith CE, Tanaka T, Cade BE, Gottlieb DJ, et al. Habitual sleep duration is associated with BMI and macronutrient intake and may be modified by CLOCK genetic variants. Am J Clin Nutr. 2015;101:135-43.

37. Prats-Puig A, Grau-Cabrera P, Riera-Perez E, Cortes-Marina R, Fortea E, Soriano-Rodriguez P, et al. Variations in the obesity genes FTO, TMEM18 and NRXN3 influence the vulnerability of children to weight gain induced by short sleep duration. Int $\mathrm{J}$ Obes. 2013;37:182-7.

38. Faraguna U, Vyazovskiy VV, Nelson AB, Tononi G, Cirelli C. A causal role for brain-derived neurotrophic factor in the homeostatic regulation of sleep. J Neurosci. 2008;28:4088-95.

39. Cirelli C, Tononi G. Differential expression of plasticity-related genes in waking and sleep and their regulation by the noradrenergic system. J Neurosci. 2000;20:9187-94.

40. Conti B, Maier R, Barr AM, Morale MC, Lu X, Sanna PP, et al. Region-specific transcriptional changes following the three antidepressant treatments electro convulsive therapy, sleep deprivation and fluoxetine. Mol Psychiatry. 2007;12:167-89.

41. Nakamura K, Fuster JJ, Walsh K. Adipokines: a link between obesity and cardiovascular disease. J Cardiol. 2014;63:250-9. 
42. Kelesidis T, Kelesidis I, Chou S, Mantzoros CS. Narrative review: the role of leptin in human physiology: emerging clinical applications. Ann Intern Med. 2010;152:93-100.

43. Willer CJ, Speliotes EK, Loos RJ, Li S, Lindgren CM, Heid IM, et al. Six new loci associated with body mass index highlight a neuronal influence on body weight regulation. Nat Genet. 2009;41:25-34.
44. Yeo GS, Farooqi IS, Aminian S, Halsall DJ, Stanhope RG, O'Rahilly S. A frameshift mutation in MC4R associated with dominantly inherited human obesity. Nat Genet. 1998;20:111-2.

45. Tung YC, Yeo GS, O'Rahilly S, Coll AP. Obesity and FTO: changing focus at a complex locus. Cell Metabol. 2014;20:710-8. 